preferentially move by sliding along them (Fig. 1d, red arrow) - resembling molecules moving on rails, as the researchers suggested. The track is only rarely changed by hopping events in the perpendicular direction (Fig. 1d, yellow arrow). Importantly, an unexpected second mode contributes to the diffusion process over the organic surface. By rotating its backbone so that it is perpendicular to the grooves, the molecule can unlock itself from the rails and undergo long jumps (Fig. 1d, green arrows). This mode is responsible for efficient and fast diffusion over several adsorption sites. In the simulated trajectories, cases were observed where up to five minima were bypassed before the adsorbate realigned and switched back into the sixth rail.
Obtaining access to such extraordinary diffusion mechanisms represents a significant achievement for the analysis of molecular dynamics. Being able to carry out the experiments at temperatures relevant for the system under investigation is the basis for further progress in the fabrication of organic devices. Moreover, the scope of the demonstrated approach extends way beyond unravelling the peculiarities of organic thin-film growth. Taking a closer look at the motion of interfacial supramolecular systems ${ }^{9}$, surface-templated chemical reactions ${ }^{10}$ and the growth of novel 2D materials ${ }^{11}$ are seemingly straightforward examples of potential extensions to this research that may reveal other unexpected and exciting insights.
Florian Klappenberger is in the Physics

Department E20, Technical University Munich, 85748 Garching, Germany.

e-mail: florian.klappenberger@tum.de

References

1. Dobson, C. M. Nature 426, 884-890 (2003).

2. Whitesides, G. M., Mathias, J. P. \& Seto, C. T. Science 254, 1312-1319 (1991).

Bhushan, B., Israelachvili, J. N. \& Landman, U. Nature 374, 607-616 (1995).

4. Barth, J. V. Surf. Sci. Rep. 40, 75-149 (2000).

5. Facchetti, A. Chem. Mater. 23, 733-758 (2011)

6. Palma, C.-A. et al. Nature Commun. 6, 6210 (2015).

7. Müllegger, S. et al. Phys. Rev. Lett. 112, 117201 (2014).

8. Rotter, P. et al. Nature Mater. 15, 397-400 (2016)

9. Bonifazi, D., Mohnani, S. \& Llanes-Pallas, A. Chem. Eur. J. 15, 7004-7025 (2009)

10. Klappenberger, F. et al. Acc. Chem. Res. 48, 2140-2150 (2015).

11. Butler, S. Z. et al. ACS Nano 7, 2898-2926 (2013).

\title{
FROM PILLAR TO POST
}

"It works in practice, but will it work in theory?" This quote is often directed as a jibe towards academic economists more concerned about their idealized mathematical models than with how things happen in the real world. But one might also be tempted to say that it characterizes the distinction between the physicist and the engineer. In this caricature, a physicist wants a solvable analytical model at the cost of too many approximations to the truth, whereas the engineer is happy with empirical rules of thumb lacking any theoretical foundation.

Of course, neither picture does justice to its discipline. But this image comes to mind when contemplating the efforts of Schellenberger et al. to understand droplet movement on superhydrophobic surfaces (Phys. Rev. Lett. 116, 096101; 2016). The researchers have used laser scanning confocal microscopy to investigate, with submicrometre resolution, the configurations at the advancing and receding edges of liquid drops on a superhydrophobic surface textured with an array of microscopic cylindrical posts and coated with hydrophobic fluoroalkyl silanes.

Hydrophobicity could (for our hypothetical physicist) seem a simple matter to understand. We could quantify it using the static, macroscopic contact angle of a water droplet on the surface: the larger the angle, the less the droplet tends to spread and the more hydrophobic the surface. For perfect hydrophobicity, the droplet beads into a sphere with a contact angle of $180^{\circ}$. This contact angle is well understood via Young's equation - it expresses the balance of interfacial tensions between solid, liquid and air.

But when a droplet moves, the situation is complicated. Then, pinning of the droplet edge can produce contact angles greater than this equilibrium value at the advancing edge and less than that at the receding edge, depending on the messy business of how pinning happens at surface defects. The matter is more complicated still for superhydrophobic surfaces, where the droplet is typically propped up on asperities on a textured surface, with air in the gaps beneath. In such cases a droplet can spontaneously roll off an inclined surface, leading to the self-cleaning 'lotus leaf effect' that makes such materials of practical interest for applications such as car windshields.

This 'propped up' configuration is called Cassie wetting, and the equilibrium contact angle is then often described by a modified form of Young's free-energy balance

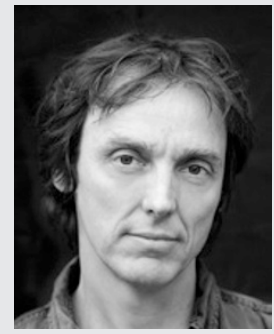

PHILIP BALL

called the Cassie-Baxter theory. But Schellenberger et al. find that the reality is at best only crudely approximated by this theory. For most superhydrophobic surfaces, they say (typically with macroscopic contact angles $>150^{\circ}$ ) the advancing edge of a droplet generally has a microscopic contact angle very close to $180^{\circ}$, and a better measure of the true superhydrophobicity is supplied by the receding angle.

The receding edge moves in a series of jumps that occur each time the microscopic contact angle there falls to some threshold value - which seems to be more or less identical to the apparent macroscopic contact angle, which in this case is about $140^{\circ}$. This, then, offers a more reliable empirical measure of the surface performance. But now the physicist might ask: can we say why this receding angle takes the value it does? 